Asim Kichloo, MD

Department of Internal Medicine, Central Michigan University College of Medicine, Saginaw, MI; Department of Internal

Medicine, Samaritan Medical Center, Watertown, NY
Zain El-Amir, MD

Department of Internal Medicine, Central Michigan

University College of Medicine, Saginaw, MI
Farah Wani, MD

Department of Family Medicine, Samaritan Medical Center, Watertown, NY

Hassan Beiz, MD

Department of Internal Medicine, Central Michigan

University College of Medicine, Saginaw, MI

\title{
Mortal remains disposal in the wake of the COVID-19 pandemic
}

\section{Posted January 21, 2021}

\section{ABSTRACT}

When dealing with infectious disease-related deaths, it is important to handle the remains of the deceased in a respectful and safe manner. There is no known evidence of SARS-CoV-2 transmission through handling of COVID-19 victim remains. However, guidelines recommend appropriate precautions to ensure safety from any potential risk. Discussions of safe and dignified postmortem care in COVID-19 cases can guide future decision making to encourage safety, dignity, and respect for all.

\section{INTRODUCTION}

Transmission of COVID-19 when handling those who have died is low. ${ }^{1}$ However, safety in handling the deceased in the COVID-19 pandemic is still important to protect the living. We discuss safe practices for handling mortal remains as well as changes in post-mortem burials and mourning practices amid the pandemic.

\section{MORTAL REMAINS DISPOSAL}

There have been no scientifically confirmed cases of COVID-19 spreading via the handling of mortal remains. ${ }^{1}$ The World Health Organization (WHO) has published guidelines regarding the handling of mortal remains during the pandemic. ${ }^{2}$ The US Centers for Disease Control and Prevention (CDC) has also commented on COVID-19 burials, with one publication comparing burials in COVID-19 and Ebola virus outbreaks. ${ }^{3}$ The CDC notes that there is little risk of acquiring COVID-19 from mortal remains

The statements and opinions expressed in COVID-19 Curbside Consults are based on experience and the available literature as of the date posted. While we try to regularly update this content, any offered recommendations cannot be substituted for the clinical judgment of clinicians caring for individual patients.

doi:10.3949/ccjm.88a.ccc070 with COVID-19 and that a team is not required for the burial process. The family may prepare the body using appropriate personal protective equipment (PPE), such as gowns, gloves, masks, goggles, face shields, as well as with diluted chlorine and/or soap and water. ${ }^{3}$

The virus is mainly spread person-to-person through respiratory droplets and fomites; infection is also possible from contact with contaminated surfaces and subsequent touching of one's own mouth, eyes, or nose. ${ }^{4}$ The virus is thought to survive several hours outside of the host but may live for days in certain conditions. ${ }^{4}$ Thus, performing any sort of aerosolgenerating procedure or one where there is a risk of contaminated fluids splashing requires appropriate protection through safety measures such as donning appropriate personal protective equipment and being rigorous about hand-washing practices.

The first priority in handling the deceased's body according to the WHO should be the safety and wellbeing of all involved. ${ }^{2}$ The WHO affirms that the dignity of the deceased and their loved ones as well as ay cultural and religious traditions should be respected throughout the process. ${ }^{4,5}$ Authorities may need to be consulted on a case-by-case basis to discuss balancing the rights of families, the need to investigate the cause of death, and the risk of exposure to the virus. ${ }^{2}$

All personnel who interact with the body should apply standard precautions, which includes the use of PPE such as gowns and gloves, in addition to hand hygiene. Face protection, including face shields and goggles, should be considered if there is a risk of exposure to bodily fluid or secretions. All lines, catheters, and tubes should be removed prior to transfer. Bodily fluids leaking from orifices should be contained prior to transport. ${ }^{2}$ Body bags are not necessary but can be used to contain fluid leakage if appropriate. ${ }^{2}$ Once 
the body arrives at the location for mortuary care, it should be handled by staff who wear appropriate PPE and take standard precautions such as donning gloves, impermeable gowns or gowns with impermeable aprons atop, medical masks, and eye protection. ${ }^{2}$

If the ceremony for the deceased is performed at home, or if mortuary services or post-mortem standard care are not readily available, those preparing the deceased should wear gloves when contacting the body. The number of individuals involved in the preparation process should be kept to a minimum. If there is a risk of bodily fluid splashing, facial protection (in the form of a face shield, goggles, and a medical mask) is recommended. Clothing worn during the body preparation should be removed and washed immediately after the process is complete. Hands should also be washed with soap and water. The body of the deceased should not be kissed by those preparing it. ${ }^{2}$ If possible, washing rituals should be replaced with spraying. ${ }^{6}$

Children and those over 60 years old or with underlying conditions should not be involved in the preparation of the body and should not interact with it directly. Those viewing the body should stay at a minimum of 1 meter away and should refrain from kissing and touching the body. Those viewing should keep at least 1 meter away from one another and wash their hands with soap and water afterwards. Those placing the body in the grave, on the funeral pyre, or in another location should wear gloves when moving the body and wash their hands with soap and water after glove removal. ${ }^{2}$ An alternative option may be to cover the body with transparent clothes to avoid direct contact with the body. ${ }^{6}$ The corpses can be disposed through cremation or burial. ${ }^{2}$ There may be local and national requirements in place directing the handling and disposition of the remains. ${ }^{2}$

The CDC has provided postmortem guidance with specific regard for the autopsy process. ${ }^{7}$ Autopsies may be performed on patients suspected to have COVID19 and in patients known to have COVID-19. In patients with suspected COVID-19, post-mortem swabs can be gathered from the upper respiratory tract with a nasopharyngeal swab or lower respiratory tract with a lung swab from each lung. ${ }^{7}$ Reverse transcription-polymerase chain reaction (RT-PCR) remains the gold standard for diagnostic detection of SARSCoV-2. In cases of confirmed COVID-19 infection, post-mortem swabs for other respiratory pathogens, such as influenza virus, and other microbiologic and infectious diseases may be indicated. Formalin-fixed tissues from major organs, such as the lungs, can be submitted and tested in some cases. ${ }^{7}$

\section{ADDITIONAL ASPECTS OF END OF LIFE CARE}

As the number of deaths has increased due to COVID19 , so too has the demand for burials and cremations, which has become especially problematic in hardhit areas. ${ }^{8}$ Crematories in New York have extended operating hours, with some reporting nearly aroundthe-clock operations to accommodate the demand. Funeral directors have struggled to find supply chains with available PPE to keep safe operations running. ${ }^{8}$

Recommendations regarding delays in grief rituals include increasing interactions while patients are still alive through modern strategies. Mobile phones and electronic tablets can be used to record and share messages with family members, which may improve the quality-of-life for patients and partially alleviate the emotional burden on families. ${ }^{9}$

Burials should take place in a timely manner. Ceremonies that do not involve burials should be postponed as long as possible until the pandemic ends. If a ceremony is held, participants should maintain physical distancing and the number of participants should be limited. Hand hygiene should be practiced. ${ }^{2}$ Post-mortem live stream services and wakes may be substituted for in-person activities as families wait for a time to mourn with in-person memorial services after the pandemic. ${ }^{3}$ Telecommunicationbased alternatives have been utilized by those who are not able to hold or choose not to hold a funeral service. ${ }^{10}$ Finally, funeral homes may wish to increase their hours of operation, if possible, to accommodate increases in demand if there is appropriate PPE. Information for funeral home workers has been published by the CDC to help guide decision making. ${ }^{11}$

\section{DISCLOSURES}

The authors report no relevant financial relationships which, in the context of their contributions, could be perceived as a potential conflict of interest.

\section{REFERENCES}

1. Rani S. A review of the management and safe handling of bodies in cases involving COVID-19. Med Sci Law 2020; 60(4):287-293. doi: 10.1177/0025802420949044

2. World Health Organization. Infection prevention and control for the safe management of a dead body in the context of COVID-19. Accessed January 7, 2021. https://apps.who.int/iris/bitstream/handle/10665/331538/WHO-COVID-19-IPC_DBMgmt-2020.1-eng.pdf

3. US Centers for Disease Control and Prevention. How are COVID19 burials different from Ebola burials? Accessed January 7, 2021. https://www.cdc.gov/coronavirus/2019-ncov/downloads/globalcovid-19/COVID19vsEbola-burial-guide.pdf

4. Finegan O, Fonseca S, Guyomarc'h P, Morcillo Mendez MD, Rodriguez Gonzalez J, Tidball-Binz M, et al. International Committee of 
the Red Cross (ICRC): General guidance for the management of the dead related to COVID-19. Forensic Sci Int Synerg 2020; 2:129-137. doi: 10.1016/j.fsisyn.2020.03.007

5. Pan American Health Organization. Leadership during a pandemic: what your municipality can do. Accessed January 7, 2021. https://www.paho.org/disasters/index.php?option=com docman\&view=download\&category_slug=tools\&alias=518-whatyour-municipality-complete-toolkit\&ltemid $=1179 \&$ lang $=e n$

6. Akwa TE. From Ebola to COVID-19: Reshaping Funerals and Burial Rites in Africa? J Health Commun 2020; 5(3):7.

7. US Centers for Disease Control and Prevention. Postmortem Guidance. Accessed January 7, 2021. https://www.cdc.gov/ coronavirus/2019-ncov/hcp/guidance-postmortem-specimens.html

8. Allen G. Funeral Homes Overwhelmed With COVID-19 Cases. Accessed January 7, 2021. https://www.npr.org/2020/04/05/826972795/ funeral-homes-overwhelmed-with-covid-19-cases

9. Ingravallo F. Death in the era of the COVID-19 pandemic. Lancet Public Health 2020; 5(5):e258. doi: 10.1016/S2468-2667(20)30079-7

10. Wallace $C L$, Wladkowski SP, Gibson A, White P. Grief During the COVID-19 Pandemic: Considerations for Palliative Care Providers. J Pain Symptom Manage 2020; 60(1):e70-e76. doi: 10.1016/j. jpainsymman.2020.04.012

11. US Centers for Disease Control and Prevention. What do funeral home workers need to know about handling decedents who had COVID-19? Accessed January 7, 2021. https://www.cdc.gov/ coronavirus/2019-ncov/community/funeral-faqs.html

Address: Asim Kichloo, MD, Associate Professor Internal Medicine, Central Michigan University College of Medicine, 1000 Houghton Avenue, Saginaw, Michigan 48602; kichlooasim@gmail.com 